\title{
Superheavy-quarkonium decays with two Higgs doublets
}

\author{
O. J. P. Éboli \\ Departamento de Física Matemática, Instituto de Física, Universidade de São Paulo, Caixa Postal 20516, \\ 01498 São Paulo, São Paulo, Brazil \\ A. A. Natale \\ Instituto de Física, Universidade Estadual Paulista, Caixa Postal 5956, 01405 São Paulo, Brazil \\ F. R. A. Simão \\ Centro Brasileiro de Pesquisas Físicas, Rua Dr. Xavier Sigaud, 150, 22290 Rio de Janeiro, Rio de Janeiro, Brazil
}

(Received 29 November 1988)

\begin{abstract}
We study the decay modes of a $S$-wave superheavy quarkonium, formed by a possible fourthgeneration quark in two-Higgs-doublet models. Because of the enhancement of Yukawa couplings and longitudinal weak bosons the main decays of these superheavy states will be into neutral scalar bosons $H_{i}^{0} H_{j}^{0}$ and a charged scalar plus a $W$ boson. If the $H^{\mp} W^{ \pm}$channel is open for the $\psi\left(1^{--}\right)$ superheavy quarkonium it will provide a quite clean signal for a charged Higgs boson. The decay of the pseudoscalar quarkonium $\eta\left(0^{-+}\right)$into a $Z$ boson and one of the scalars will also be present in a large amount.
\end{abstract}

\section{INTRODUCTION}

The standard model of strong and electroweak interactions achieved remarkable experimental success, although it contains a replication of families and a mass-generation mechanism whose fundamental origin remains uncertain. Exactly these two aspects may provide the most simple extensions of this model; i.e., we can have a fourth family and (or) a second Higgs doublet, in addition to the one necessary for mass generation. Within the present experimental status these two possibilities can be easily accommodated in the theory.

If a fourth fermionic family is found it will show an extremely rich phenomenology. In particular, the decays of quarkonium states formed by a down-type quark of a fourth-generation doublet have been quite extensively studied recently in the standard one-Higgs-doublet model. ${ }^{1,2}$ In Refs. 1 and 2, Barger et al. showed that for these superheavy quarkonium states new channels will be open, such as $W^{+} W^{-}, Z Z$, and $Z \gamma$ with large branching fractions. They also observed that the decay of the pseudoscalar state into a $Z$ boson plus a Higgs boson will be a quite clear signal for the Higgs particle. ${ }^{3}$

In this paper we extend the analysis of Refs. 1 and 2 to the two-Higgs-doublet case, and, as will be shown, the introduction of this extra doublet will change considerably the picture of heavy-quarkonium decays. We shall analyze only the $S$-wave states, i.e., $\eta\left(0^{-+}\right)$and $\psi\left(1^{--}\right)$resonances, since their decays will contain most of the modes which can occur in a two-doublet model and they will be present at a significant level. The main decays will be those involving at least one scalar boson $\left(H_{i}^{0} H_{j}^{0}\right.$, $H^{ \pm} W^{\mp}, H^{0} Z$, and $\left.H^{+} H^{-}\right)$.

In the two-doublet model the $\psi$ decay channel into $H^{ \pm} W^{\mp}$ is enhanced by the coupling of the heavy quarks to the Higgs boson and to the longitudinal $W$, and it dominates over the $W W, Z Z$, and $H Z$ modes, being one of the best possibilities of detecting a charged Higgs boson, as long as we find a heavy fourth-generation $d$-type quark. The channel $H^{0} H^{0}$, which in the one-doublet model is absent as a decay channel of $\eta$ and $\psi$, is now open for $H_{i}^{0} H_{3}^{0}(i=1,2)$, due to the presence of the pseudoscalar $H_{3}^{0}$. In this last case the branching fractions will show a large dependence on the variation of the many parameters of the model, and for simplicity most of our numerical results will be obtained for the minimal supersymmetric model.

The content of our paper is distributed as follows. Section II contains a few tools about quarkonium states that shall be used in the subsequent sections. In Sec. III we briefly review some aspects of the two-doublet model and its reduction to the minimal supersymmetric case. The calculation of the $\eta$ and $\psi$ quarkonium partial widths and branching fractions will be displayed in Sec. IV. In Sec. $\mathrm{V}$ we discuss our results and present our conclusions about superheavy-quarkonium decays with two Higgs doublets.

\section{REMARKS ON SUPERHEAVY QUARKONIUM PHYSICS}

In this section we collect the main expressions of quarkonium physics that will be used throughout the paper, and we are deliberately brief in our presentation since these aspects have been discussed extensively in Ref. 2, to which we refer the reader interested in further details.

Initially we recall that the decay widths will depend on the quarkonium potential, or, more precisely, on its wave function. For the $S$-wave quarkonium the squared wave function at the origin is determined from the potential as

$$
\left|R_{S}(0)\right|^{2}=m_{Q}\left\langle\frac{d V}{d r}\right\rangle
$$


and we shall consider only the Coulomb potential in our calculations. Therefore,

$$
\left|R_{S}(0)\right|^{2}=4\left(\frac{2}{3} \alpha_{s} m_{Q}\right)^{3},
$$

where $m_{Q}$ is the fourth-generation $d$-type quark mass, and $\alpha_{s}$ is the running strong coupling at the quarkonium scale

$$
\alpha_{s}=\frac{12 \pi}{\left(33-2 N_{f}\right) \ln \left(m_{Q}^{2} / \Lambda^{2}\right)},
$$

with $N_{f}=6$ and $\Lambda=0.12 \mathrm{GeV}$ (Ref. 2).

For a very heavy quarkonium the short-distance behavior, given by the Coulomb potential, will certainly dominate, and neglecting the long-range forces present in more realistic potentials, such as the Cornell, Richardson, and Wisconsin ones, will not introduce large errors at least for the $S$ states. As discussed in Ref. 2 we shall be only slightly underestimating $\left|R_{S}(0)\right|^{2}$. There are other sources of uncertainties in our calculation, which include the nonintroduction of relativistic corrections to the quarkonium potential and the QCD corrections, which modify the wave function at origin and the amplitude calculation. These approximations produce different effects, but considering that we are underestimating the wave function at origin when using the Coulomb potential, we expect that at the end we shall have a reasonable lower bound for the partial widths.
The decay amplitudes for the $\eta$ and $\psi$ states can be obtained in a standard fashion: $:^{4,2}$

$$
\begin{aligned}
& A(\eta)=\left(\frac{3}{16 \pi M_{\eta}}\right)^{1 / 2} R_{S}(0) \operatorname{Tr}\left[\phi_{F} \gamma_{5}\left(-\varnothing+m_{\eta}\right)\right], \\
& A(\psi)=-\left(\frac{3}{16 \pi M_{\psi}}\right)^{1 / 2} R_{S}(0) \operatorname{Tr}\left[\phi_{F} \boldsymbol{\epsilon}\left(-\varnothing+m_{\psi}\right)\right],
\end{aligned}
$$

where $\phi_{F}$ is the scattering matrix element for $q+\bar{q} \rightarrow X$ ( $X$ is the final state), $Q$ is the quarkonium fourmomentum, and $\epsilon$ is the polarization vector of the spinone quarkonium. From (2.4) and (2.5) the decay rates are easily obtained.

\section{TWO-DOUBLET MODELS}

In this section we will introduce the basic couplings of two-doublet models which will be used in the subsequent section. We consider models where each doublet gives mass to one specific quark, i.e., up-type or down-type quarks, as is necessary in supersymmetric models, as well as for suppression of flavor-changing neutral currents.

Some of the couplings that we shall need are the ones between gauge and scalar bosons, and the Higgs-boson self-couplings. The first ones are determined uniquely by gauge invariance (see Refs. 5 and 6), and the trilinear Higgs-boson couplings come from the potential ${ }^{5,6}$

$$
\begin{aligned}
V\left(\phi_{1}, \phi_{2}\right)= & \lambda_{1}\left(\phi_{1}^{\dagger} \phi_{1}-v_{1}^{2}\right)^{2}+\lambda_{2}\left(\phi_{2}^{\dagger} \phi_{2}-v_{2}^{2}\right)^{2}+\lambda_{3}\left[\left(\phi_{1}^{\dagger} \phi_{1}-v_{1}^{2}\right)+\left(\phi_{2}^{\dagger} \phi_{2}-v_{2}^{2}\right)\right]^{2}+\lambda_{4}\left[\left(\phi_{1}^{\dagger} \phi_{1}\right)\left(\phi_{2}^{\dagger} \phi_{2}\right)-\left(\phi_{1}^{\dagger} \phi_{2}\right)\left(\phi_{2}^{\dagger} \phi_{1}\right)\right] \\
& +\lambda_{5}\left[\operatorname{Re}\left(\phi_{1}^{\dagger} \phi_{2}\right)-v_{1} v_{2} \cos \xi\right]^{2}+\lambda_{6}\left[\operatorname{Im}\left(\phi_{1}^{\dagger} \phi_{2}\right)-v_{1} v_{2} \sin \xi\right]^{2},
\end{aligned}
$$

where $v_{1}$ and $v_{2}$ are the vacuum expectation values of the neutral components of the scalar doublets:

$$
\left\langle\phi_{1}\right\rangle=\left(\begin{array}{c}
v_{1} \\
0
\end{array}\right),\left\langle\phi_{2}\right\rangle=\left(\begin{array}{c}
v_{2} e^{i \xi} \\
0
\end{array}\right) \text {. }
$$

The phase $\xi$ can be removed from the potential with a redefinition of $\phi_{2}\left(\rightarrow e^{i \xi} \phi_{2}\right)$. After the symmetry breaking we have $M_{W}=(1 / \sqrt{2}) g v$ where $v^{2}=v_{1}^{2}+v_{2}^{2}$. The physical Higgs bosons are obtained from the diagonalization of the mass matrix and are given by

$$
\begin{aligned}
& H^{ \pm}=-\phi_{1}^{ \pm} \sin \beta+\phi_{2}^{ \pm} \cos \beta, \\
& H_{1}^{0}=\sqrt{2}\left[\left(\operatorname{Re} \phi_{1}^{0}-v_{1}\right) \cos \alpha+\left(\operatorname{Re} \phi_{2}^{0}-v_{2}\right) \sin \alpha\right], \\
& H_{2}^{0}=\sqrt{2}\left[\left(-\operatorname{Re} \phi_{1}^{0}-v_{1}\right) \sin \alpha+\left(\operatorname{Re} \phi_{2}^{0}-v_{2}\right) \cos \alpha\right], \\
& H_{3}^{0}=\sqrt{2}\left(-\operatorname{Im} \phi_{1}^{0} \sin \beta+\operatorname{Im} \phi_{2}^{0} \cos \beta\right),
\end{aligned}
$$

where

$$
\begin{aligned}
& \tan \beta=\frac{v_{2}}{v_{1}}, \\
& \sin 2 \alpha=\frac{2 v_{1} v_{2}\left(4 \lambda_{3}+\lambda_{5}\right)}{\left\{\left[v_{1}^{2}\left(4 \lambda_{1}+4 \lambda_{3}-\lambda_{5}\right)+v_{2}^{2}\left(\lambda_{5}-4 \lambda_{2}-4 \lambda_{3}\right)\right]^{2}+4\left(\lambda_{3}+\lambda_{5}\right) v_{1}^{2} v_{2}^{2}\right\}^{1 / 2}} .
\end{aligned}
$$

The trilinear Higgs-boson couplings $\left(H_{i} H_{j} H_{k}\right)$ are obtained from (3.1) with the help of (3.2) and (3.3). There are some constraints on the couplings of (3.1) coming from partial-wave unitarity ${ }^{7}$ but they are quite loose, and basically we escape from them if the Higgs-boson masses are lighter than $1 \mathrm{TeV}$. There are some other constraints from low-energy physics; ${ }^{8}$ however, we continue to have a large range of variation for the many parameters. 
Therefore, in the next section, the trilinear Higgs-boson couplings will be denoted by $\lambda_{i j k}$ (representing the coupling $H_{i} H_{j} H_{k}$ ), although the numerical results will be given for the supersymmetric Lagrangian

$$
\begin{aligned}
\mathcal{L}_{H_{i} H_{j} H_{k}}=- & g M_{W} H^{+} H^{-}\left[H_{1}^{0} \cos (\beta-\alpha)+H_{2}^{0} \sin (\beta-\alpha)\right] \\
- & \frac{g M_{z}}{4 \cos \theta_{W}}\left[H_{1}^{0} \cos (\beta+\alpha)+H_{2}^{0} \sin (\beta+\alpha)\right] \\
& \times\left\{\cos 2 \alpha\left[\left(H_{1}^{0}\right)^{2}-\left(H_{2}^{0}\right)^{2}\right]-2 H_{1}^{0} H_{2}^{0} \sin 2 \alpha\right. \\
& \left.-\left[\left(H_{3}^{0}\right)^{2}+\left(2 H^{+} H^{-}\right)\right] \cos 2 \beta\right\} .
\end{aligned}
$$

In Sec. IV the coupling $i \lambda_{133}$, for example, has been set equal to $i\left(g M_{Z} / 2 \cos \theta_{W}\right) \cos 2 \beta \cos (\beta+\alpha)$ when performing the numerical calculation of partial widths. Following this supersymmetric reduction of the Higgs potential we also have constrained the possible variation of masses by the relations: ${ }^{6}$

$$
M_{H_{3}^{0}}^{2}=M_{H^{ \pm}}^{2}-M_{W}^{2}
$$

$$
\begin{aligned}
& M_{H_{1}^{0}}^{2}=M_{H_{3}^{0}}^{2}+M_{Z}^{2}-M_{H_{2}^{0}}^{2}, \\
& \tan 2 \alpha=\tan 2 \beta\left(\frac{M_{H_{1}^{0}}^{2}+M_{H_{2}^{0}}^{2}}{M_{H_{3}^{0}}^{2}-M_{Z}^{2}}\right), \\
& \tan 2 \beta=\operatorname{sgn}\left(v_{2}-v_{1}\right)\left(\frac{\left(M_{H_{1}^{0}}^{2}-M_{Z}^{2}\right)\left(M_{Z}^{2}-M_{H_{2}^{0}}^{2}\right)}{M_{H_{1}^{0}}^{2} M_{H_{2}^{0}}^{2}}\right)^{1 / 2},
\end{aligned}
$$

and we shall assume that $\operatorname{sgn}\left(v_{2}-v_{1}\right)=+1$ is the favored sector, since it emerges naturally in models with $m_{t}>m_{b}$. Notice that for the supersymmetric case, we may obtain a numerical result that is much smaller than the one we would obtain in a standard two-doublet model, where the trilinear couplings can be considerably larger, as happens in a strongly interacting Higgs sector.

We will also need the couplings of the quarks with the Higgs bosons, which are given by

$$
\begin{aligned}
\mathcal{L}_{q q^{\prime} H}= & \frac{g}{2 \sqrt{2} M_{W}}\left\{H^{+} \bar{t}^{\prime}\left[\left(m_{b^{\prime}} \tan \beta+m_{t^{\prime}} \cot \beta\right)+\left(m_{b^{\prime}} \tan \beta-m_{t^{\prime}} \cot \beta\right) \gamma_{5}\right] b^{\prime}+\text { H.c. }\right\} \\
& -\frac{g m_{b^{\prime}}}{2 M_{W} \cos \beta}\left[\bar{b}^{\prime} b^{\prime}\left(H_{1}^{0} \cos \alpha-H_{2}^{0} \sin \alpha\right)-i \bar{b}^{\prime} \gamma_{{ }_{5}} b^{\prime} H_{3}^{0} \sin \beta\right],
\end{aligned}
$$

where $\left(t^{\prime}, b^{\prime}\right)$ is the fourth-generation doublet and $m_{t^{\prime}\left(b^{\prime}\right)}$ their masses. The couplings of charged Higgs boson with the quarks involve the Kobayashi-Maskawa matrix, which was not introduced in (3.8), because models of mass (mixing) generation with four families of quarks suggest that $\left|K_{b^{\prime} t^{\prime}}\right|^{2} \approx 1$ and for the intergeneration angles $\left|K_{b^{\prime} q}\right|^{2}<<1$ (Ref. 9). Therefore, for our purposes, this will be a minor approximation.

\section{DECAY RATES}

This section contains the decay rate calculation of superheavy quarkonia ( $\eta$ and $\psi$ ) within a two-doublet model. ${ }^{10}$ Initially we shall make some comments on singlequark and annihilation decays and secondly we detail the other decays.

\section{A. Single-quark decay}

The decay $b^{\prime} \rightarrow q+W^{ \pm}$has been analyzed in Ref. 2, and it is proportional to the mixing angle $\left|K_{b^{\prime} q}\right|^{2}$, as noticed previously this angle may be quite small, ${ }^{9}$ resulting in a branching ratio for this particular process negligible when compared with the main decays. In the twodoublets model there is another contribution to be considered, $b^{\prime} \rightarrow q+H^{ \pm}$, but the same small mixing angle comes into play turning also this event negligible. ${ }^{11}$

\section{B. $\gamma \gamma, g g, g g g, f f$}

The annihilation decays into photons or gluons are exactly the same as in Ref. 2:

$$
\begin{aligned}
& \Gamma(\eta \rightarrow \gamma \gamma)=\frac{12 \alpha^{2} e_{b^{\prime}}^{4}}{M^{2}}\left|R_{S}(0)\right|^{2}, \\
& \Gamma(\eta \rightarrow g g)=\frac{8 \alpha_{s}^{2}}{3 M^{2}}\left|R_{S}(0)\right|^{2}, \\
& \Gamma(\psi \rightarrow 3 g)=\frac{40\left(\pi^{2}-9\right) \alpha_{S}^{3}}{81 \pi M^{2}}\left|R_{S}(0)\right|^{2},
\end{aligned}
$$

where $M$ is the $\eta$ or $\psi$ mass, and $e_{b^{\prime}}$ is the $b^{\prime}$ quark charge in units of the proton charge. In the two-doublet model the new neutral bosons couple to a fermion-antifermion $f \bar{f}$ pair; however, these scalar $S$ channels do not contribute to $\eta(\psi) \rightarrow f \bar{f}$ and again the result is the same as in Ref. 2:

$$
\begin{aligned}
\Gamma(\eta \rightarrow f \bar{f})= & \frac{3 \alpha_{Z}^{2} N_{f} \beta_{f}}{32} \frac{M_{f}^{2}}{M_{Z}^{4}}\left|R_{S}(0)\right|^{2}, \\
\Gamma(\psi \rightarrow f \bar{f})= & \frac{4 \alpha^{2} N_{f} \beta_{f}}{M^{2}} \\
& \times\left[( 1 + 2 R _ { f } ) \left[e_{b^{\prime}}^{2} e_{f}^{2}+\frac{2 e_{b^{\prime}} e_{f} v_{b^{\prime}} v_{f}}{x_{W}\left(1-x_{W}\right)} \frac{1}{1-R_{Z}}\right.\right. \\
& \left.\quad+\frac{v_{b^{\prime}}^{2} v_{f}^{2}}{x_{W}^{2}\left(1-x_{W}\right)^{2}} \frac{1}{\left(1-R_{Z}\right)^{2}}\right] \\
& \left.\quad+\beta_{f}^{2} \frac{v_{b^{\prime}}^{2} a_{f}^{2}}{x_{W}^{2}\left(1-x_{W}\right)^{2}} \frac{1}{\left(1-R_{Z}\right)^{2}}\right]\left|R_{S}(0)\right|^{2},
\end{aligned}
$$


where $x_{W}=\sin ^{2} \theta_{W}, \alpha_{Z}=\alpha / x_{W}\left(1-x_{W}\right), N_{f}$ is a color factor $\left(N_{q}=3, \quad N_{l}=1\right), \quad R_{i}=M_{i}^{2} / M^{2} \quad(i=f, Z, H, W$, $\left.b^{\prime}, t^{\prime}\right), \beta_{f}=\left(1-4 R_{f}\right)^{1 / 2}$ is the fermion velocity in the quarkonium rest frame, and $v_{i}$ and $a_{i}$, the vector and axial-vector coupling of the fermion $i=f$ or $b^{\prime}$ to the $Z$, are given by

$$
v_{i}=\frac{1}{2}\left(I_{3 L}+I_{3 R}\right)-e_{i} \sin ^{2} \theta_{W}, a_{i}=\frac{1}{2}\left(I_{3 L}-I_{3 R}\right),
$$

where $I_{3 L}\left(I_{3 R}\right)$ is the third component of the weak isospin for the left- (right-)handed fermion.

\section{C. $\mathrm{H}_{i}^{0} \mathrm{H}_{j}^{0}$}

In the standard model the $\psi$ state is not allowed to decay into two Higgs bosons $\left(H^{0} H^{0}\right)$, and the same holds for $\eta$ as long as $C P$ symmetry is conserved. In the twodoublet model the following partial widths are zero:

$$
\begin{aligned}
& \Gamma\left(\eta \rightarrow H_{i}^{0} H_{i}^{0}\right)=0, \quad i=1,2,3 \\
& \Gamma\left(\eta \rightarrow H_{1}^{0} H_{2}^{0}\right)=0, \\
& \Gamma\left(\psi \rightarrow H_{i}^{0} H_{i}^{0}\right)=0, \quad i=1,2,3 \\
& \Gamma\left(\psi \rightarrow H_{1}^{0} H_{2}^{0}\right)=0 .
\end{aligned}
$$

Bose symmetry forbids the channel $H_{i}^{0} H_{i}^{0}$ and $C P$ invariance the decay into $H_{1}^{0} H_{0}^{2}$; however, due to the presence

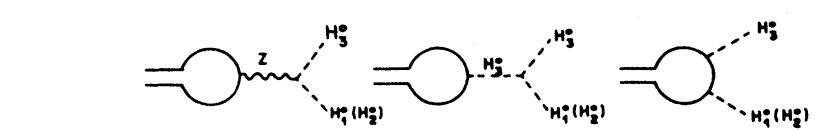

(a)
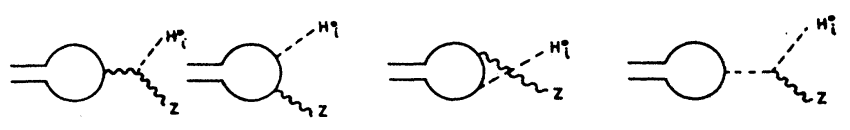

(b)

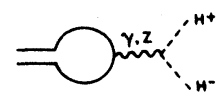

(c)
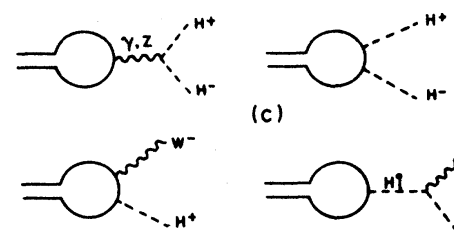

(c)

(d)

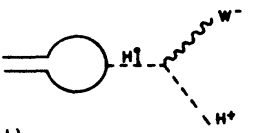

FIG. 1. (a) Contributions to $\eta \rightarrow H_{3}^{0} H_{1}^{0}(i=1,2)$, only the first diagram contributes to $\psi \rightarrow H_{3}^{0} H_{1}^{0}$; (b) contributions to $\eta \rightarrow H_{i}^{0} Z$ (first and fourth diagrams) and $\psi \rightarrow H_{i}^{0} Z$ (except the last one); (c) diagrams contributing to $\psi \rightarrow H^{+} H^{-}$; (d) diagrams contributing to $\eta$ and $\psi \rightarrow H^{ \pm} W^{\mp}$.

of the pseudoscalar $H_{3}^{0}$ we can have the decays $\eta \rightarrow H_{3}^{0}+H_{1(2)}^{0}$ and $\psi \rightarrow H_{3}^{0}+H_{1(2)}^{0}$; the diagrams of the contributions to these decays are shown in Fig. 1 and the partial width for the $\eta$ decay is

$$
\begin{aligned}
\Gamma\left(\eta \rightarrow H_{1}^{0} H_{3}^{0}\right)=\frac{3 \alpha_{Z}^{2}}{16 M^{2}} \lambda^{1 / 2}\left(1, R_{H_{3}}, R_{H_{1}}\right) & {\left[-\frac{a_{b^{\prime}}}{2} \sin (\alpha-\beta) \frac{R_{H_{3}}-R_{H_{1}}}{R_{Z}}+\frac{2 \sqrt{1-x_{W}}}{g} \lambda_{33 i}\left[\frac{R_{b^{\prime}}}{R_{Z}}\right]^{1 / 2} \frac{\tan \beta}{M} \frac{1}{1-R_{H_{3}}}\right.} \\
& \left.-4 \frac{R_{b^{\prime}}}{R_{Z}} \frac{\cos \alpha}{\cos \beta} \tan \beta \frac{2 R_{b^{\prime}}^{1 / 2}+R_{H_{3}}-R_{H_{1}}}{2 R_{H_{3}}+2 R_{H_{1}}-1-4 R_{b^{\prime}}}\right]^{2}\left|R_{S}(0)\right|^{2}
\end{aligned}
$$

where $\lambda_{33 i}(i=1,2)$ is the trilinear Higgs-boson coupling $\left(H_{3}^{0} H_{3}^{0} H_{i}^{0}\right)$, which in the supersymmetric case is

$$
\begin{aligned}
& \lambda_{331}=\frac{g}{2 \sqrt{1-x_{W}}} M_{Z} \cos 2 \beta \cos (\alpha+\beta), \\
& \lambda_{332}=\frac{-g}{2 \sqrt{1-x_{W}}} M_{Z} \cos 2 \beta \sin (\alpha+\beta),
\end{aligned}
$$

and the function $\lambda(x, y, z)=(x-y-z)^{2}-4 y z$. The partial width of $\eta \rightarrow H_{2}^{0} H_{3}^{0}$ is obtained from (4.10) with the replacements

$$
\begin{aligned}
& \sin (\alpha-\beta) \rightarrow \cos (\alpha-\beta), \\
& M_{H_{1}} \rightarrow M_{H_{2}}, \\
& \lambda_{331} \rightarrow \lambda_{332}, \\
& \cos \alpha \rightarrow-\sin \alpha .
\end{aligned}
$$

Notice that this $\eta$ decay will be enhanced not only by the large Yukawa couplings, but also, in nonsupersymmetric models, by a possible strong trilinear Higgs-boson coupling.
The $\psi$ decay into $H_{3}^{0} H_{1}^{0}$ has a partial width given by

$$
\begin{aligned}
\Gamma\left(\psi \rightarrow H_{3}^{0} H_{1}^{0}\right)= & \frac{\alpha_{Z}^{2} v_{b^{\prime}}^{2} \sin ^{2}(\alpha-\beta)}{64 M^{2}} \lambda^{1 / 2}\left(1, R_{H_{1}}, R_{H_{3}}\right) \\
& \times \frac{\left(1-2 R_{H_{3}}-2 R_{H_{1}}\right)+\left(R_{H_{3}}-R_{H_{1}}\right)^{2}}{\left(1-R_{Z}\right)^{2}} \\
& \times\left|R_{S}(0)\right|^{2}
\end{aligned}
$$

only the first diagram of Fig. 1(a) contributes in this case.

The width $\Gamma\left(\psi \rightarrow H_{2}^{0} H_{3}^{0}\right)$ is obtained from Eq. (4.12) making the change

$$
M_{H_{1}} \rightarrow M_{H_{2}}, \quad \sin ^{2}(\alpha-\beta) \rightarrow \cos ^{2}(\alpha-\beta)
$$

\section{D. $\boldsymbol{Z H}_{i}^{0}$}

This is one of the most interesting superheavy quarkonium decays in the one-doublet model, since it can give a quite clear signal for the Higgs boson. ${ }^{1-3}$ In the twodoublet model the diagrams contributing to $\mathrm{ZH}_{i}^{0}$ decay are depicted in Fig. 1(b). The last diagram of this figure 
does not appear in the one-doublet model, and together with the first one it is responsible for the decay $\eta \rightarrow H_{1(2)}^{0} Z$. The partial width is

$$
\begin{aligned}
& \Gamma\left(\eta \rightarrow H_{i}^{0} Z\right)=\frac{3 \alpha_{Z}^{2}}{16 M_{Z}^{2}} \lambda^{1 / 2}\left(1, R_{H_{i}}, R_{Z}\right) \\
& \times\left(-1+\frac{\left(1+R_{Z}-R_{H_{i}}\right)^{2}}{4 R_{Z}}\right) \\
& \times\left(-a_{b^{\prime}, X_{i}}+\tan \beta h_{i} \frac{1}{1-R_{H_{3}}}\right]^{2}\left|R_{S}(0)\right|^{2},
\end{aligned}
$$

where we assumed $2 m_{b^{\prime}} \approx M$. Setting $i=1$ or 2 we obtain $\Gamma$ for $H_{1}^{0} Z$ or $H_{2}^{0} Z$ in the final state, with

$$
\begin{aligned}
& X_{1}=h_{2}=\cos (\beta-\alpha), \\
& X_{2}=-h_{1}=\sin (\beta-\alpha) .
\end{aligned}
$$

The partial width for the pseudoscalar boson $\left(\eta \rightarrow Z H_{3}^{0}\right)$ is zero in a $C P$-conserving theory. The $\psi \rightarrow Z H_{i}^{0}(i=1,2)$ widths are

$$
\begin{aligned}
\Gamma\left(\psi-Z H_{i}^{0}\right)= & \frac{\alpha_{Z}^{2} v_{b^{\prime}}^{2}}{16 M^{2}} \lambda^{1 / 2}\left(1, R_{H_{i}}, R_{Z}\right) \\
& \times\left[\frac{X_{i}^{2}}{4\left(1-R_{z}\right)^{2}}\left(8 R_{Z}+\Delta^{2}\right)\right. \\
& +\frac{Y_{i}^{2}}{2 R_{Z}\left(1-R_{H_{i}}-R_{Z}\right)^{2}}\left(2 R_{Z}+\Delta^{2}\right) \\
& \left.+\frac{3 X_{i} Y_{i} \Delta}{\left(1-R_{Z}\right)\left(1-R_{Z}-R_{H_{i}}\right)^{2}}\right]\left|R_{S}(0)\right|^{2},
\end{aligned}
$$

where $i=1,2$, with the $X_{i}$ given by Eq. (4.14), $\Delta=\left(1+R_{Z}-R_{H_{i}}\right)$, and

$$
\begin{aligned}
& Y_{1}=-\cos \alpha / \cos \beta, \\
& Y_{2}=\sin \alpha / \cos \beta .
\end{aligned}
$$

The above result agrees with the one of Ref. 3 .

For the $\psi$ decay into the pseudoscalar $H_{3}^{0}$ and a $Z$ we have

$$
\begin{aligned}
& \Gamma\left(\psi \rightarrow H_{3}^{0} Z\right)= \frac{\alpha_{Z}^{2} v_{b^{\prime}}^{2} \tan ^{2} \beta}{2 M^{2}} \lambda^{1 / 2}\left(1, R_{Z}, R_{H_{3}}\right) \\
& \times \frac{R_{b^{\prime}}}{R_{Z}}\left[\frac{\left(1+R_{Z}-R_{H_{3}}\right)^{2}-4 R_{Z}}{\left(2 R_{Z}+2 R_{H_{3}}-1-4 R_{b^{\prime}}\right)^{2}}\right] \\
& \times\left|R_{S}(0)\right|^{2}, \\
& \text { E. } \gamma H_{i}^{0}
\end{aligned}
$$

Because of $C$ conservation we have only the $\psi$ decaying into $\gamma H_{i}^{0}$. This decay in the one-doublet model was first computed by Wilczek, ${ }^{12}$ and the production of the pseudoscalar $H_{3}^{0}$ in toponium decay $\left(t t^{\prime}\right) \rightarrow H_{3}^{0} \gamma$ has also been discussed recently in the two-doublet model ${ }^{13,3}$ and in a superstring model. ${ }^{10}$

The partial widths of $\psi \rightarrow H_{i} \gamma(i=1,2)$ are given by

$$
\begin{aligned}
\Gamma\left(\psi \rightarrow \gamma H_{i}^{0}\right)= & \frac{8 \alpha^{2} e_{b^{\prime}}^{2} Y_{i}^{2}}{x_{W}\left(1-x_{W}\right) M^{2}} \lambda^{1 / 2}\left(1,0, R_{H_{i}}\right) \\
& \times \frac{R_{b^{\prime}}}{R_{Z}} \frac{\left(2 R_{b^{\prime}}^{1 / 2}-R_{H_{i}}\right)^{2}}{\left(2 R_{H_{i}}-1-4 R_{b^{\prime}}\right)^{2}}\left|R_{S}(0)\right|^{2},
\end{aligned}
$$

where $Y_{i}$ was defined in Eq. (4.16). The decay with the pseudoscalar in the final state is

$$
\begin{aligned}
\Gamma\left(\psi \rightarrow \gamma H_{3}^{0}\right)= & \frac{8 \alpha^{2} e_{b^{\prime}}^{2} \tan ^{2} \beta}{x_{W}\left(1-x_{W}\right) M^{2}} \lambda^{1 / 2}\left(1, R_{H_{3}}, 0\right) \\
& \times \frac{R_{b^{\prime}}}{R_{Z}} \frac{\left(1-R_{H_{3}}\right)^{2}}{\left(2 R_{H_{3}}-1-4 R_{b^{\prime}}\right)^{2}}\left|R_{S}(0)\right|^{2} .
\end{aligned}
$$

$$
\text { F. } \boldsymbol{H}^{+} \boldsymbol{H}^{-}
$$

The heavy $\eta$ quarkonium is not allowed to decay into $\mathrm{H}^{+} \mathrm{H}^{-}$, and the $\psi$ widths are calculated from the diagrams of Fig. 1(c). (There is another possible diagram, exchanging neutral scalar bosons, that leads to a vanishing amplitude.) The result is

$$
\begin{aligned}
& \Gamma\left(\psi \rightarrow H^{+} H^{-}\right)= \frac{1}{16 \pi M^{2}} \beta_{H^{+}}^{3} \\
& \times\left[4 \alpha e_{b^{\prime}}+\frac{\alpha_{Z} v_{b^{\prime}} \cos 2 \theta_{W}}{2\left(1-R_{Z}\right)}\right. \\
&\left.\quad-2 \frac{\alpha_{Z}}{R_{Z}}\left(r^{2}+t^{2}\right) \frac{1}{1+4 R_{t^{\prime}}-4 R_{H^{+}}}\right)^{2} \\
& \quad \times\left|R_{S}(0)\right|^{2},
\end{aligned}
$$

where

$$
\begin{aligned}
& r=R_{b^{\prime}}^{1 / 2} \tan \beta+R_{t^{\prime}}^{1 / 2} \cot \beta, \\
& t=R_{b^{\prime}}^{1 / 2} \cot \beta-R_{t^{\prime}}^{1 / 2} \tan \beta,
\end{aligned}
$$

and $\beta_{H^{+}}=\left(1-4 R_{H^{+}}\right)^{1 / 2}$ is the $H^{+}$velocity in the quarkonium rest frame.

$$
\text { G. } \boldsymbol{H}^{ \pm} \boldsymbol{W}^{\mp}
$$

In this case there are two diagrams, which are displayed in Fig. 1(d), that contribute to the decay of $\eta$ and $\psi$. This will be one of the most interesting decays in this model, since it is enhanced by the longitudinal $W$ and by the large Yukawa coupling. The partial width for $\eta \rightarrow H W$ is 


$$
\begin{aligned}
\Gamma\left(\eta \rightarrow H^{ \pm} W^{\mp}\right)= & \frac{3}{32} \alpha_{Z}^{2} \lambda^{3 / 2}\left(1, R_{W}, R_{H^{+}}\right) \\
& \times \frac{M^{2}}{M_{Z}^{4}}\left[\frac{1}{2} \frac{p}{q}+\frac{\tan \beta}{l}\right]^{2}\left|R_{S}(0)\right|^{2},
\end{aligned}
$$

where

$$
\begin{aligned}
& p=(t-r)+2 R_{t^{\prime}}^{1 / 2}(r+t), \\
& q=\left(R_{W}+R_{H^{+}}-\frac{1}{2}-2 R_{t^{\prime}}\right), \\
& l=\left(1-R_{H_{3}}\right),
\end{aligned}
$$

and $r(t)$ is defined in Eq. (4.21a) [(4.21b)]. Finally the width for $\psi \rightarrow H W$ is given by

$$
\begin{aligned}
\Gamma\left(\psi \rightarrow H^{ \pm} W^{\mp}\right)= & \frac{2}{\pi^{2} M^{4} q^{2}} \lambda^{1 / 2}\left(1, R_{W}, R_{H^{+}}\right) \\
\times & \left(\frac{3+\rho}{4} \delta^{2}\right. \\
& \quad-M^{2}\left(1+R_{W}^{2}-R_{H}^{2}\right)(r-t) \rho \delta \\
& \left.+4(r-t)^{2} M_{W}^{2}(\rho+2) \rho\right)\left|R_{S}(0)\right|^{2},
\end{aligned}
$$

where

$$
\begin{aligned}
& \delta=2(r-t)\left(2 R_{t^{\prime}}+R_{W}-R_{H}\right), \\
& \rho=-1+\frac{1}{4} \frac{1}{R_{W}}\left(1+R_{W}^{2}-R_{H^{+}}^{2}\right)^{2},
\end{aligned}
$$

with $r$ and $t$ given by Eqs. (4.21) and $q$ by Eq. (4.23b).

\section{H. $W W, Z Z, Z_{\gamma}$}

We must also consider the quarkonium decays into gauge-boson pairs $(W W, Z Z$, and $Z \gamma)$. The possible new contributions to these decays in a two-doublet model vanish for the $\eta$ and $\psi$ states. Therefore we obtained the very same results as the one-doublet model contained in Ref. 2, to which we refer the reader for further details.

\section{RESULTS AND CONCLUSIONS}

The branching ratios and widths for the superheavy $\eta$ and $\psi$ decays in the two-doublet model are shown in Figs. 2-6. The numerical results were obtained for the minimal supersymmetric model for which the relations (3.7) are valid, and we used $M_{W}=82.5 \mathrm{GeV}$ and $\sin ^{2} \theta_{W}=0.226$.

As the mass relations (3.7) are not maintained in the nonsupersymmetric models we may have a quite large range of masses for the scalar bosons, and most of the decays, and in particular $\eta(\psi) \rightarrow H_{i}^{0} H_{j}^{0}$, could be modified due to a larger or smaller phase space for the decay products. The only decay that may deviate largely from the supersymmetric case, when we maintain the angles $\alpha$ and $\beta$ fixed, is $\eta(\psi) \rightarrow H_{3}^{0} H_{i}^{0}(i=1,2)$ due to a completely free parameter in the coupling $H_{3}^{0} H_{3}^{0} H_{i}^{0}$. Furthermore, if we do have a supersymmetric model we could have some other competitive channels only for sufficiently light supersymmetric scalar partners.

The superheavy $\eta$ decays will be mostly dominated by the decays into $H_{3}^{0} H_{2}^{0}$ and $H_{3}^{0} H_{1}^{0}$, which provide approximately $80 \%$ of the decays. The decays into $H_{1}^{0} Z$ will be responsible for $10 \%$ of the decays, and in a smaller amount we will have decays into $H^{\mp} W^{ \pm}$and $H_{2}^{0} Z$. Notice from Figs. 2(a) -2 (d) that increasing the chargedHiggs-boson mass (the $H_{3}^{0}$-boson mass also increases), the fraction of decays into $\mathrm{H}_{3}^{0} \mathrm{H}_{i}^{0}$ decreases in favor of $\mathrm{ZH}_{1}^{0}$ and $H^{ \pm} W^{\mp}$. These last decays are enhanced by one large Yukawa coupling and the longitudinal $(Z$ and $W)$ boson, and the channels into $H_{3}^{0} H_{i}^{0}(i=1,2)$ are enhanced by two large Yukawa couplings; whether one or another decay is more important depend on the mass parameters. For the $\psi$ quarkonium we see from Figs. 3(a) $-3(d)$ that as long as the channel $H^{ \pm} W^{\mp}$ is open it dominates completely the $\psi$ decays, with a secondary contribution from $H^{+} H^{-}$. In these calculations we assumed $m_{t^{\prime}}=m_{b^{\prime}}$, and we will comment on this approximation in the sequence.

TABLE I. Number of events expected at the SSC per years for the decays $\eta \rightarrow H_{1}^{0} H_{3}^{0}$, $\eta(\psi) \rightarrow H^{ \pm} W^{\mp}$, and $\psi \rightarrow H^{+} H^{-}$. The quarkonium masses have been chosen to be 250,500 , and 750 $\mathrm{GeV}$. The first entry for each decay corresponds to a charged-Higgs-boson mass $M_{H^{+}}=100 \mathrm{GeV}$ and the second to $M_{H^{+}}=250 \mathrm{GeV}$ (with $M_{H_{2}^{0}}=50 \mathrm{GeV}$ ).

\begin{tabular}{lccc}
\hline$M_{Q \bar{Q}}(\mathrm{GeV})$ & 250 & 500 & 750 \\
\hline$\eta \rightarrow H_{1}^{0} H_{3}^{0}$ & $10^{5}$ & $2 \times 10^{3}$ & $8 \times 10^{2}$ \\
& 0 & $6 \times 10^{3}$ & $4.2 \times 10^{3}$ \\
$\eta \rightarrow H^{ \pm} W^{\mp}$ & $4 \times 10^{3}$ & $3 \times 10^{2}$ & $1.3 \times 10^{2}$ \\
& 0 & $1.4 \times 10^{3}$ & $7 \times 10^{2}$ \\
$\psi \rightarrow H^{ \pm} W^{\mp}$ & $1.2 \times 10^{4}$ & 250 & 50 \\
& 0 & 425 & 80 \\
& $2 \times 10^{3}$ & 200 & 40 \\
& 0 & 0 & 17 \\
\hline
\end{tabular}


Concerning the $\eta$ decays, the channels into gluons ( $g g$ ), top quarks $(t \bar{t})$ turn out to be negligible as soon as the ones into $H_{3}^{0} H_{i}^{0}, H_{i}^{0} Z$, and $H^{ \pm} W^{+}$are open. The radiative and hadronic transitions will always be quite small. The same holds for $\psi$ decays, where the dominant decays in an one-doublet model were into $W W$ and $Z Z$. With a fourth-generation lepton $\left(l^{\prime}\right)$ there would be a possible decay $\eta(\psi) \rightarrow l^{\prime} \bar{l}^{\prime}$, although it is not strong enough to change the results of Figs. 2 and 3, unless all the scalar

(a)

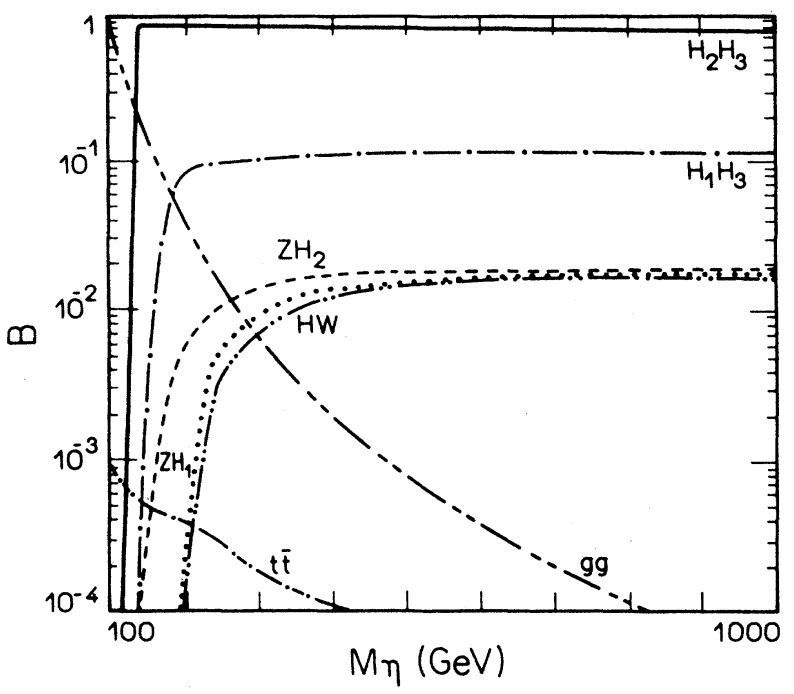

(c)

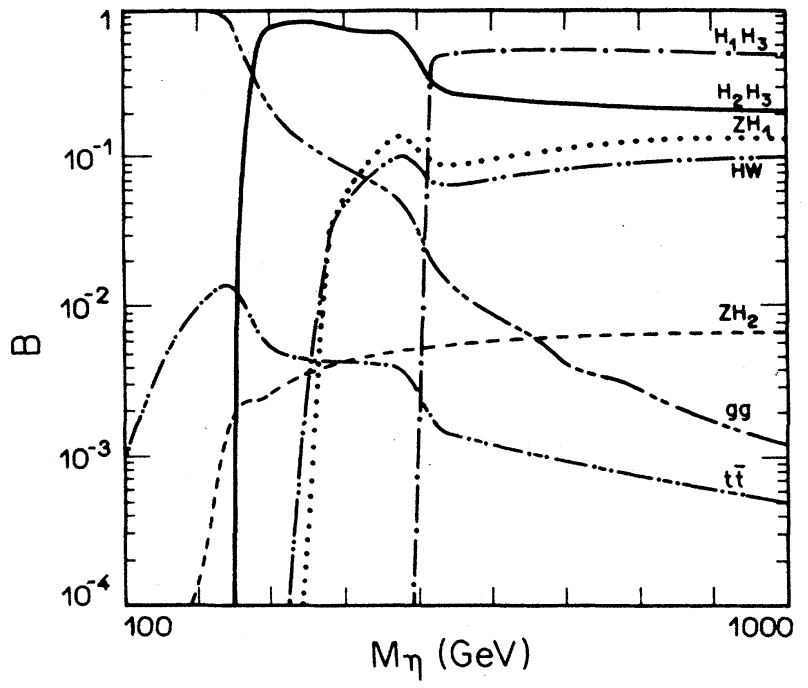

channels are closed, which is not a plausible possibility, at least in a supersymmetric case when one of the scalars is lighter than the $Z$. It is clear that if we do not have supersymmetry and if all the scalar sector is very heavy, the superheavy quarkonium decays will be mostly the ones discussed in Ref. 2.

It is interesting to notice that in the case of toponium decays, as quoted by Varger and Whisnant, ${ }^{11}$ the decay into $H^{ \pm} W^{\mp}$ is suppressed by the ratio $m_{b} / m_{t}$. This does

(b)

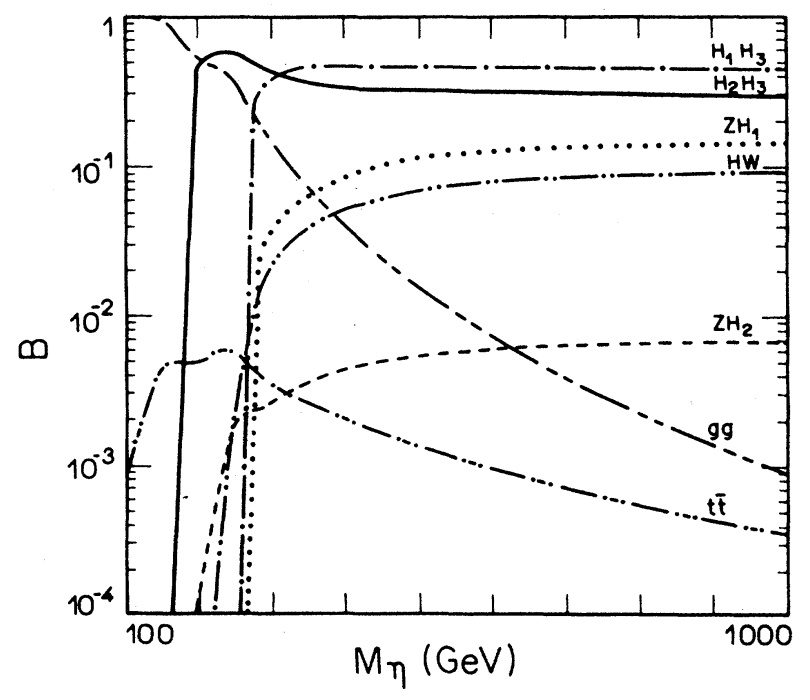

(d)

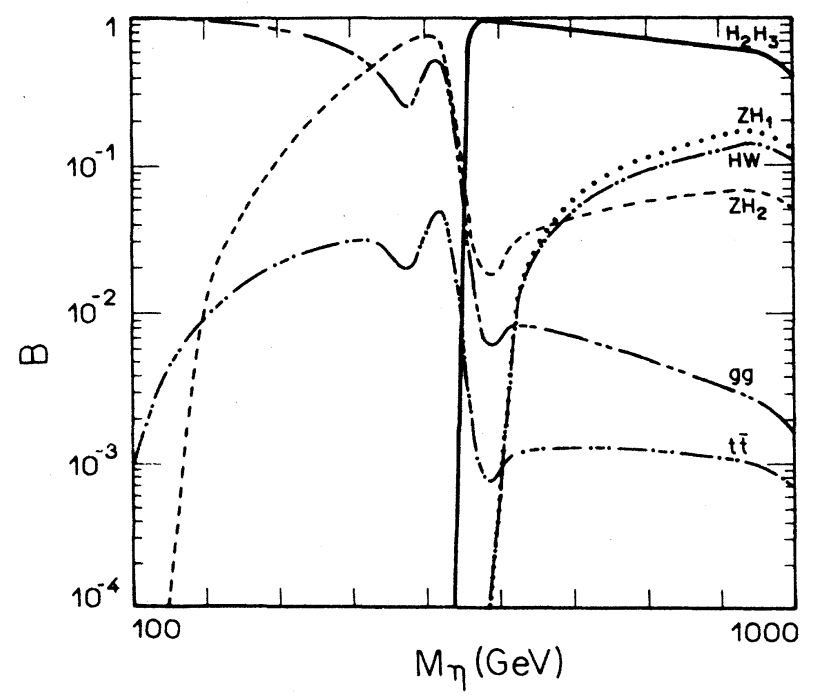

FIG. 2. The branching ratios for $\eta$ decays versus the quarkonium mass for $M_{H_{2}}=50 \mathrm{GeV}$ and (a) $M_{H^{+}}=100 \mathrm{GeV}$, (b) $M_{H^{+}}=150$ $\mathrm{GeV}$, (c) $M_{H^{+}}=250 \mathrm{GeV}$, (d) $M_{H^{+}}=500 \mathrm{GeV}$. 
not happen for a fourth generation where the ratio $m_{b^{\prime}} / m_{t^{\prime}}$ is not expected to be small. ${ }^{9}$ We have computed the $\eta \rightarrow H^{\mp} W^{ \pm}$decay for different values of the mass splitting $\Delta m=m_{t^{\prime}}-m_{b^{\prime}}$, and the results are shown in Fig. 4 , where only a minor change in the branching ratio can be seen.

The total widths for $\eta$ and $\psi$ are shown in Figs. 5 and

(a)

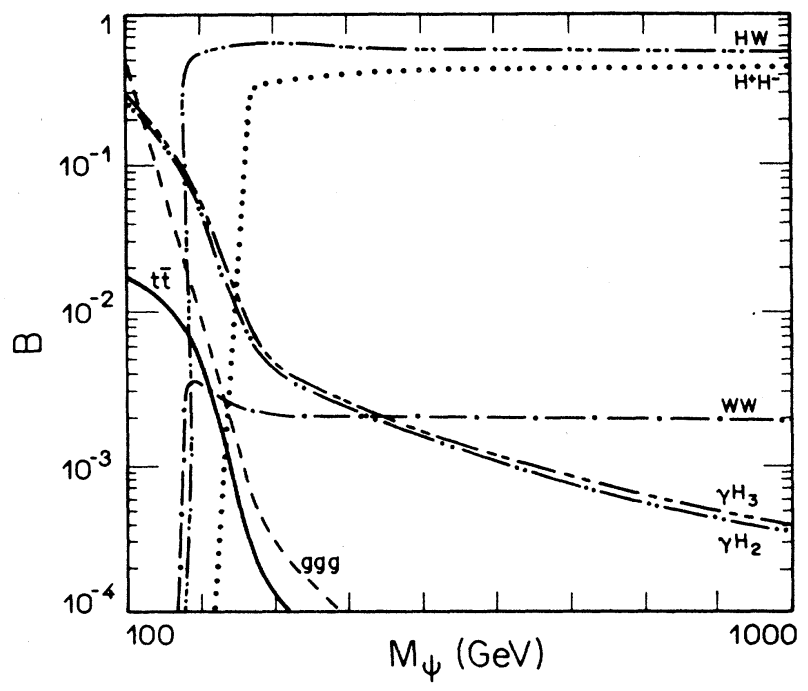

(c)

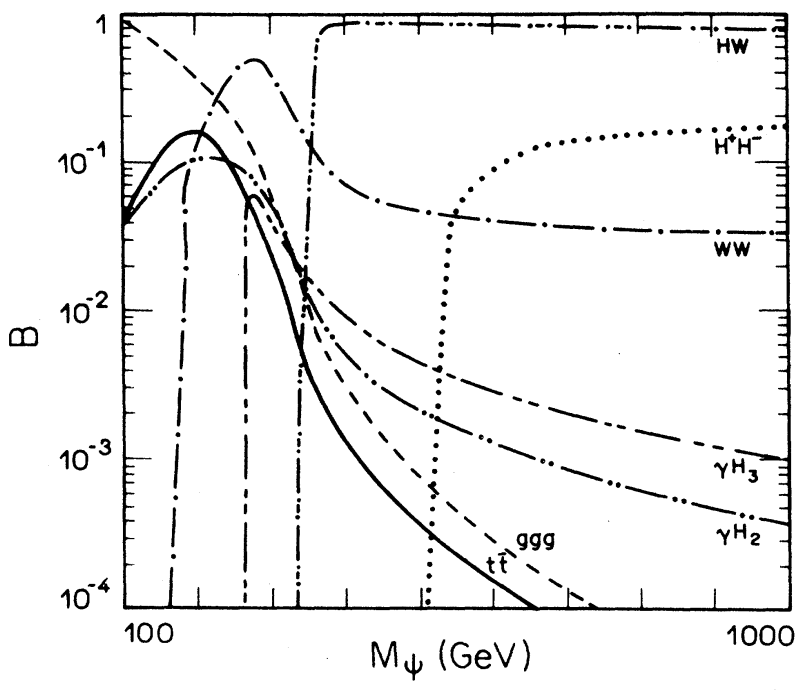

6, respectively, which may be of several $\mathrm{GeV}$ for large quarkonium masses. Notice that they show a large variation with the charged-Higgs-boson mass, and consequently, due to (3.7), with $M_{H_{3}}$. If these bosons are light enough, we shall have quite large cross sections for $H_{3}^{0} H_{i}^{0}$ and $\boldsymbol{H}^{ \pm} W^{\mp}$ production at multi-TeV colliders, such as

(b)

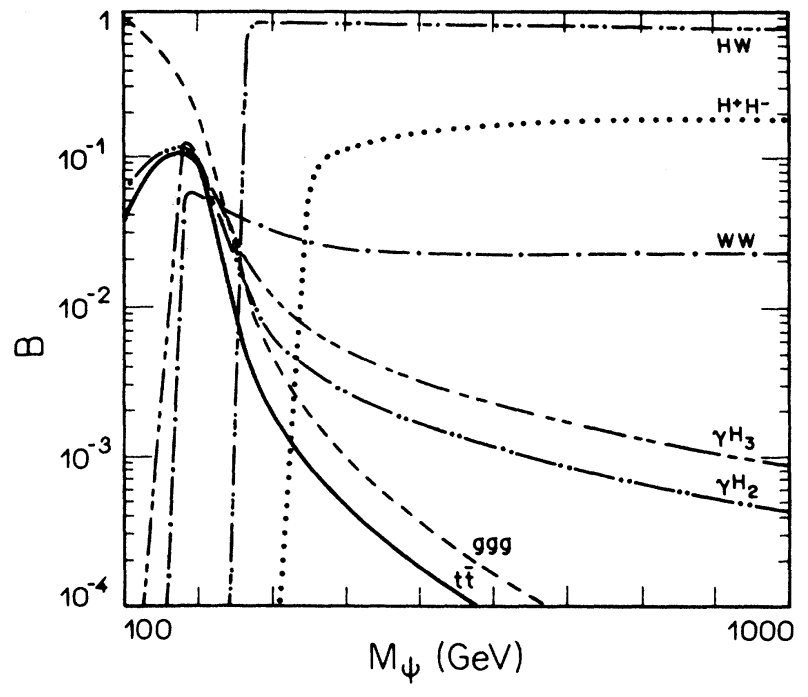

(d)

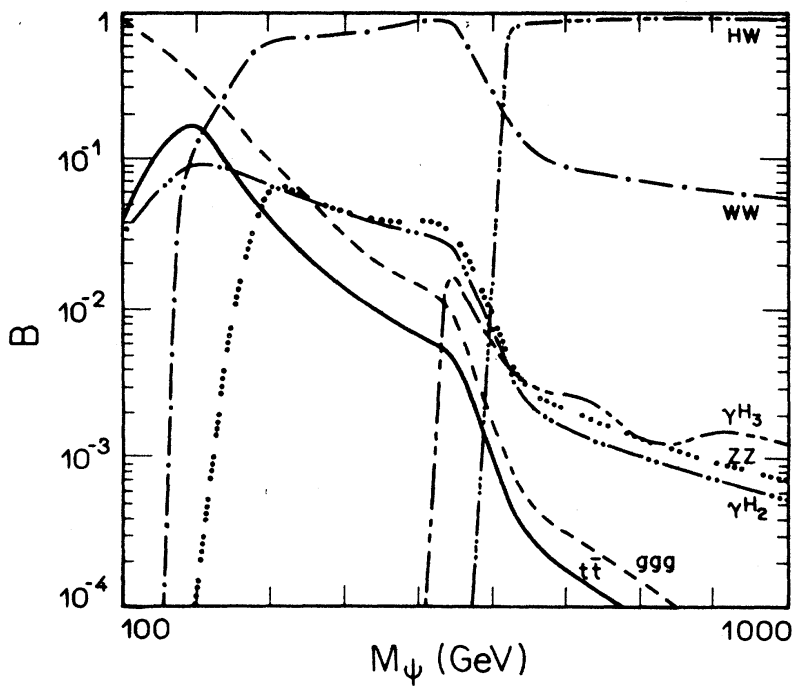

FIG. 3. The branching ratios for $\psi$ decays versus the quarkonium mass for $M_{H_{2}}=50 \mathrm{GeV}$ and (a) $M_{H^{+}}=100 \mathrm{GeV}$, (b) $M_{H^{+}}=150$ $\mathrm{GeV}$, (c) $M_{H^{+}}=250 \mathrm{GeV}$, (d) $M_{H^{+}}=500 \mathrm{GeV}$. 


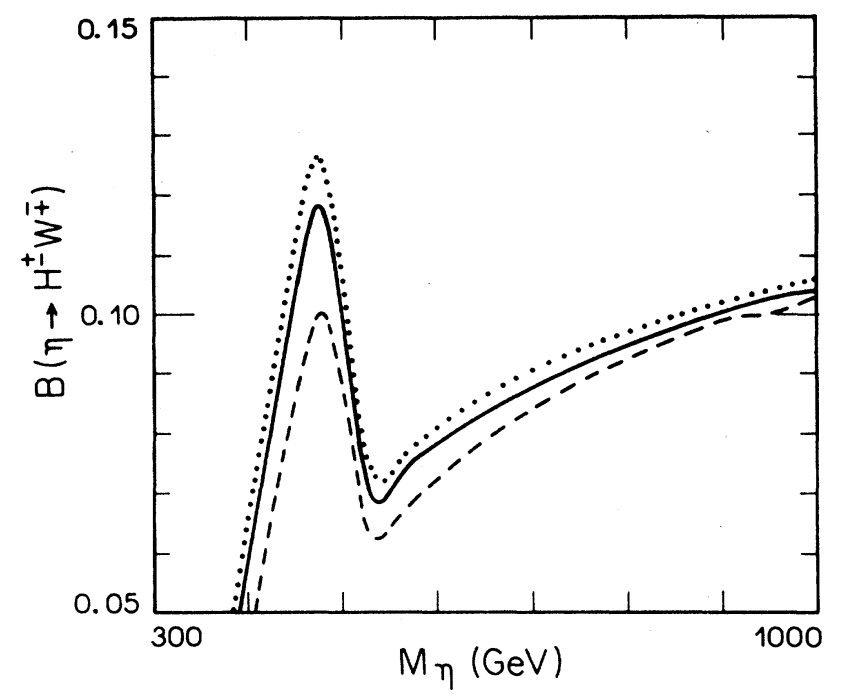

FIG. 4. The branching ratio $\eta \rightarrow H^{ \pm} W^{\mp}$ versus the quarkonium mass for $M_{H^{+}}=150 \mathrm{GeV}, M_{H_{2}}=50 \mathrm{GeV}$ and assuming (a) $m_{t^{\prime}}=m_{b^{\prime}}$ (dotted line), (b) $m_{t^{\prime}}-m_{b^{\prime}}=100 \mathrm{GeV}$ (solid line), (c) - $m_{t^{\prime}}-m_{b^{\prime}}=200 \mathrm{GeV}$ (dashed line).

the hadronic collider, the Superconducting Super Collider (SSC), or the $e^{+} e^{-}$machine, the CERN Linear Collider (CLIC).

The superheavy $\eta$ and $\psi$ mesons can be produced in a hadronic collider, such as the SSC, in a proportion roughly of order $10^{2}: 1$, respectively; therefore, certain decay modes which have moderately small branching ratios for the $\eta$ meson (up to $1 \%$ ), but are almost responsible

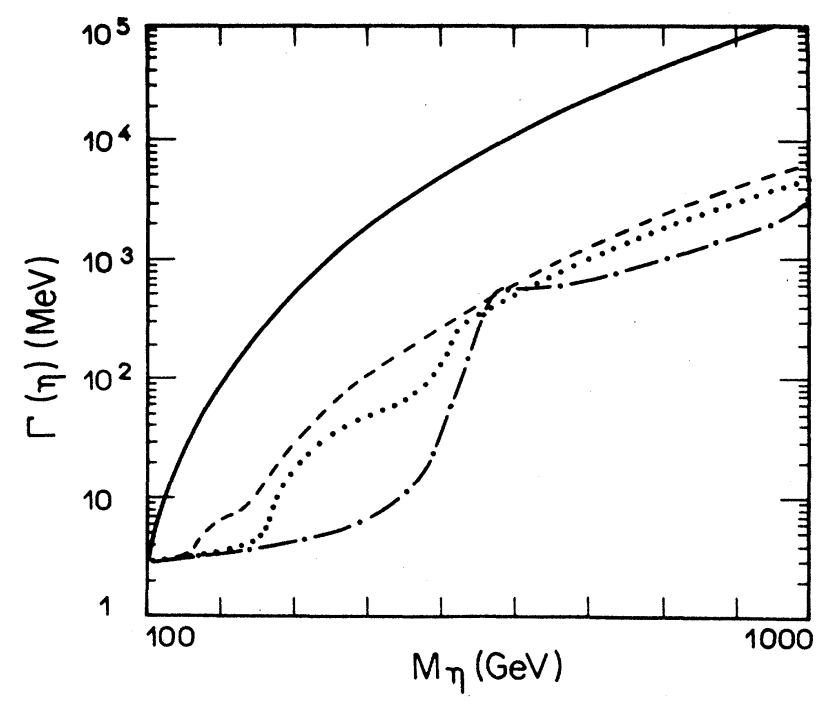

FIG. 5. The total width of the $\eta$ versus the quarkonium mass for $M_{H_{2}}=50 \mathrm{GeV}$ and (a) $M_{H^{+}}=100 \mathrm{GeV}$ (solid line), (b) $M_{H^{+}}=150 \mathrm{GeV}$ (dashed line), (c) $M_{H^{+}}=250 \mathrm{GeV}$ (dotted line); (d) $M_{H^{+}}=500 \mathrm{GeV}$ (dot-dashed line).

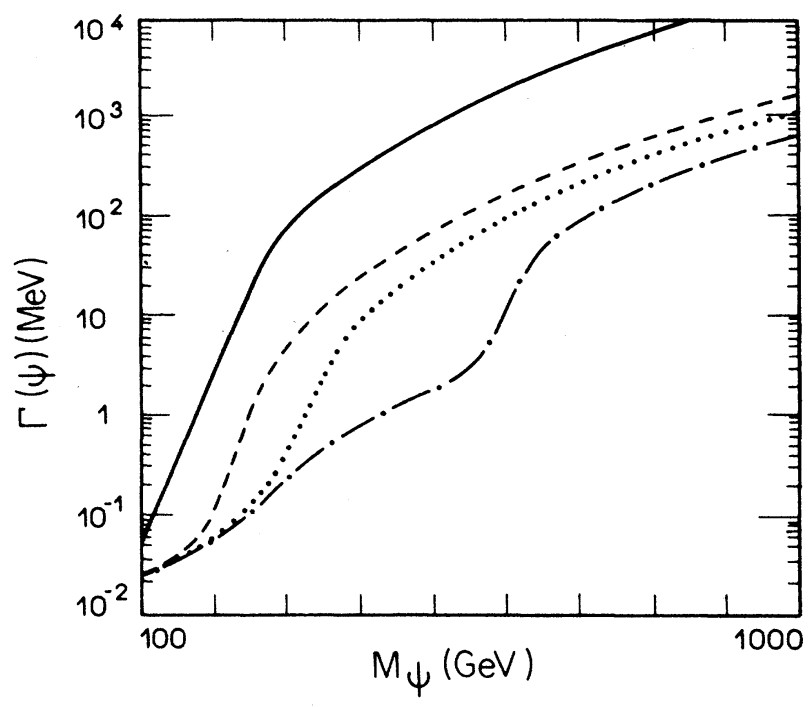

FIG. 6. The total width of the $\psi$ versus the quarkonium mass for $M_{H_{2}}=50 \mathrm{GeV}$ and (a) $M_{H^{+}}=100 \mathrm{GeV}$ (solid line), (b) $M_{H^{+}}=150 \mathrm{GeV}$ (dashed line), (c) $M_{H^{+}}=250 \mathrm{GeV}$ (dotted line); (d) $M_{H^{+}}=500 \mathrm{GeV}$ (dot-dashed line).

for the full width of the $\psi$ quarkonium, will come out with the same rate from these two states. However in one $e^{+} e^{-}$machine we shall basically produce $\psi$ 's in a quite large resonance. In each case the superheavy quarkonium will be a copious source of the scalar bosons predicted in a two-doublet model. In Table I we give a crude estimate of the number of events expected in the proposed SSC, with an integrated luminosity of $10^{4}$ $\mathrm{pb}^{-1} / \mathrm{yr}$, for the modes $H_{1}^{0} H_{3}^{0}, H^{ \pm} H^{\mp}$, and $H^{+} H^{-}$.

In $e^{+} e^{-}$colliders the Higgs bosons predicted by a two-doublet model will be mainly produced in the $\psi$ quarkonium decay. The main decay, as already seen, will be into $H^{ \pm} W^{\mp}$. As we do not have tree-level coupling $Z^{0}$ (or $\gamma$ ) $H^{ \pm} W^{\mp}$ (Ref. 14), any other possible channel producing $H^{\mp} W^{ \pm}$will have quite a small cross section; therefore, the observation of the charged scalar boson may be done if a good efficiency of detecting the $W^{ \pm}$boson is achieved; in this case the $W$ boson can be used to tag the event and its $p_{T}$ distribution shall indicate the value of $M_{H^{ \pm}}$. A good way to observe a light $H^{ \pm}$is through its decay $H^{ \pm} \rightarrow \tau^{ \pm} v$ (Ref. 15); however, once we consider a very heavy charged Higgs boson, it will mostly decay into $(t \bar{b})$, or in the pair $l^{\prime} v^{\prime}$ of the fourth generation, if this channel is open.

For a heavy charged boson the production of pairs $\mathrm{H}^{+} \mathrm{H}^{-}$via $\gamma$ and $\mathrm{Z}$ in the channel is small; therefore, production through a possible superheavy quarkonium will be the most important way to detect them in an $e^{+} e^{-}$collider.

The branching ratios of $\psi$ into one scalar plus a vector boson are small and such a final state will be immersed in a large continuum production [that is, $\left.e^{+} e^{-} \rightarrow Z \rightarrow H_{1}^{0}\left(H_{2}^{0}\right) Z\right]$.

In the case of hadronic colliders the fusion of gluons through the triangle and box diagrams, will give large 
contributions to the production of scalar-boson pairs, $Z^{0} H_{i}^{0}$, and $H^{\mp} W^{ \pm}$, on the other hand the quark and vector-boson fusion process will not be efficient to produce these same final states. ${ }^{16}$ The signals for the $H Z$ events are the same discussed by Barger et al. ${ }^{12}$ In the case of $H^{ \pm} W^{\mp}$ we could use the $W^{ \pm}$to tag the event, while the charged Higgs boson would originate two jets (probably made of $t \bar{b}$ ). Again, the observation of this event will depend on the efficiency of reconstructing the $W$ bosons.

The detection of pairs $H_{3}^{0} H_{i}^{0}$ produced in the $\eta$ quarkonium decay will not be an easy task. A large amount of the events will be materialized in four jets or four gauge bosons (this will depend obviously on the scalarboson masses), in any case the QCD background will be enormous, difficulting the observation of Higgs-boson pairs.

Our main intention in this work was to show that in a model with two Higgs doublets, the decay of a superheavy quarkonium composed by new quarks of a fourth generation, will be considerably modified in comparison with its decays in a one-doublet model. Channels such as $H_{1}^{0} Z, H_{2}^{0} Z$, and $H^{ \pm} W^{\mp}$ may be seen in the quarkonium decay, but we can expect that channels as $H_{3}^{0} H_{i}^{0}$ shall be lost in the background.

If there is a fourth generation its signal in one hadronic collider, such as the SSC, will be probably found looking for different kind of events as, for example, multilepton events. ${ }^{17}$ If this extra generation is confirmed, then we must make a deeper study of the superheavy quarkonium decays, including background, since these may be a useful tool to look for the existence of new scalar bosons.
${ }^{1}$ V. Barger et al., Phys. Rev. Lett. 57, 1672 (1986).

${ }^{2}$ V. Barger et al., Phys. Rev. D 35, 3366 (1987).

${ }^{3}$ Heavy-quarkonium decay into $H Z$ has also been studied by L. Bergstrom and P. Poutiainen, Phys. Lett. B 182, 83 (1986).

${ }^{4}$ J. H. Kühn, J. Kaplan, and E. G. Safiani, Nucl. Phys. B157, 125 (1979); B. Guberina, J. H. Kühn, R. Peccei, and R. Rückl, ibid. B174, 317 (1980).

${ }^{5}$ H. E. Haber, G. L. Kane, and T. Sterling, Nucl. Phys. B161, 493 (1979); N. G. Deshpande and E. Ma, Phys. Rev. D 18, 2574 (1978); J. F. Donoghue and L.-F. Li, ibid. 19, 945 (1979).

${ }^{6} \mathrm{~J}$. F. Gunion and H. E. Haber, Nucl. Phys. B272, 1 (1986).

${ }^{7}$ H. Hüffel and G. Pöcsik, Z. Phys. C 8, 13 (1981); for the supersymmetric case, see R. Casalbuoni, D. Dominici, F. Feruglio, and R. Gatto, Phys. Lett. B 191, 409 (1987).

${ }^{8}$ L. F. Abbot, P. Sikivie, and M. B. Wise, Phys. Rev. D 21, 1393 (1980); G. Athanasiu and F. Gilman, Phys. Lett. 153B, 274 (1985); G. Athanasiu, P. J. Franzini, and F. Gilman, Phys. Rev. D 32, 3010 (1985); see also S. Bertolini, Nucl. Phys. B 272, 77 (1986).

${ }^{9}$ V. Barger, H. Baer, K. Hagiwara, and R. J. N. Phillips, Phys. Rev. D 30, 947 (1984); T. P. Cheng and L.-F. Li, ibid. 34, 226 (1986).

${ }^{10}$ When finishing this work we came across the work of $\mathrm{V}$.
Barger and K. Whisnant, Int. J. Mod. Phys. A 3, 1907 (1988), which contains results parallel to ours, but dealing particularly with toponium decays in a superstring-inspired model.

${ }^{11}$ The single-quark decay would be important only if the heavy channels that we are considering were not open; see R. W. Robinett, Phys. Rev. D 33, 736 (1986).

${ }^{12}$ F. Wilczek, Phys. Rev. Lett. 39, 1304 (1977).

${ }^{13}$ G. Gamberini, G. F. Giudice, and G. Ridolfi, Phys. Lett. B 196, 527 (1987); Nucl. Phys. B292, 237 (1987).

14J. A. Grifols and A. Mendez, Phys. Rev. D 22, 1725 (1980).

15J. F. Gunion et al., Nucl. Phys. B294, 621 (1987).

${ }^{16}$ The production of Higgs-boson pairs through gluon fusion (compared to quark fusion) can be seen in S. D. Willenbrock, Phys. Rev. D 35, 173 (1987); O. J. P. Éboli, G. C. Marques, S. F. Novaes, and A. A. Natale, Phys. Lett. B 197, 269 (1987); R. Foot, H. Lew, and G. C. Joshi, Phys. Rev. D 37, 3161 (1988). The production of pairs $H^{0} H^{0}, H^{+} H^{-}$, and $H^{ \pm} W^{\mp}$ through vector-boson fusion was discussed by W.-Y. Keung, Mod. Phys. Lett. A 2, 765 (1987), and O. J. P. Éboli et al., Phys. Rev. D 37, 837 (1988).

${ }^{17}$ H. Baer, V. Barger, H. Goldberg, and J. Ohnemus, Phys. Rev. D 38, 3467 (1988). 